\title{
A Time-varying Observer for Linear Systems with Asynchronous Discrete-Time Measurements
}

\author{
Antonino Sferlazza and Luca Zaccarian
}

\begin{abstract}
In this paper we propose a time-varying observer for a linear continuous-time plant with asynchronous discrete-time measurements. The proposed observer is contextualized in the hybrid systems framework providing an elegant setting for the proposed solution. In particular some theoretical tools are provided, in terms of LMIs, certifying asymptotic stability of a certain compact set where the estimation error is zero. Moreover the case of asynchronous measurements is considered, i.e. when the measurements are not provided in well defined time instants, but they occur at an arbitrary time in a certain time interval. A design procedure based on the numerical solution of an infinite-dimensional LMI is also proposed, leading to a time-varying observer gain. Finally a numerical example shows the effectiveness of the proposed approach.
\end{abstract}

\section{INTRODUCTION}

In the last years, the design of observers for systems with sampled measurements has received great attention. This interest is motivated by many engineering applications, such as sampled-data systems, quantized systems, networked systems, localization of mobile vehicles, etc. [7], [16], [13]. In these cases, the output is available only at sampling instants, and, for this reason, classical observer structures cannot be used. This problem is not new in control engineering. In particular it has been considered in a stochastic framework, and particular Kalman filters have been developed for these purposes. For example in [23], a Kalman filter with intermittent observations is developed starting from the discrete Kalman filtering formulation, and modeling the input of the observation as a random process. A similar approach is followed in [18] where the observations are available according to a Bernoulli process. Further convergence analysis and boundedness analysis on the estimation error have been recently analyzed in [14] and [21].

A deterministic approach has been followed in other works. For example an interesting approach is proposed in [19] where the finite time convergence of an observer is proven for linear systems with sampled measurements, and subsequently in [20] and [4], where a similar method has been proposed for nonlinear Lipschitz systems with sampled measurements. This approach has been extended in [8] by means of new conditions in terms of linear matrix inequalities (LMIs). Moreover similar types of observers have been developed, such as in [9], where the same category of systems has been treated, but obtaining conditions in a different form, or in [17] where nonlinear uniformly observable systems are addressed. Finally, by using Lyapunov tools adapted to impulsive systems, some classes of systems with both sampled and delayed outputs are addressed in [2] and [1].

Recently, different approaches have been proposed using the hybrid system formalism of [12]. The use of the hybrid formalism provides a natural setting for the modeling of this type of observers, where both continuous-time and discretetime dynamics coexist. Indeed a sampled-data observer can be modeled by a "flow map", which describes the continuous-time dynamics when the measurement is not available, while the measurement can be considered as a discrete event and can be modeled by a suitable "jump map". This kind of formalism is applied in [10], [11], where the estimation of the state of a linear time invariant systems is proposed, with asynchronous measurements and a constant output error injection gain. In the same context [15] proposes a hybrid observer for linear systems in a distributed fashion over networks allowing only intermittent transmission.

In this paper we fit our observer in the same hybrid systems framework, but differently from the other papers presented in literature, we propose a design procedure based on numerical solution of an infinite-dimensional LMI, leading to a timevarying observer gain. In particular we provide some theoretical tools, in terms of LMIs, certifying asymptotic stability of a certain compact set where the estimation error is zero. Moreover we consider the case of asynchronous measurements, i.e. when the measurements are not provided in well defined time instants, but they occur at an arbitrary time in a certain time interval. A numerical example shows the effectiveness of the proposed approach.

Due to space constraints the proofs are omitted, but can be found in the extended version of this draft available at [22].

\section{Problem Statement}

In this work we consider a class of systems described by the following equation:

$$
\dot{x}=A x+B u,
$$

A. Sferlazza is with the CNRS, LAAS, 7 av. du Colonel Roche, F-31400, Toulouse, France. antonino.sferlazza@laas. fr

L. Zaccarian is with the CNRS, LAAS, 7 av. du Colonel Roche, F-31400, Toulouse, France, and with the Dipartimento di Ingegneria Industriale, University of Trento, 38122 Trento, Italy. zaccarian@laas.fr, luca.zaccarian@unitn.it

Research supported by ANR project LimICoS contract number 12 BS03 00501 and grant PowerLyap funded by CaRiTRo. 
where $x \in \mathbb{R}^{n}$ is the state of the system, $u:[0, \infty) \rightarrow \mathbb{R}^{q}$ is a known input that belongs to the class of locally bounded measurable functions, $A \in \mathbb{R}^{n \times n}$, and $B \in \mathbb{R}^{n \times q}$. Let us assume that an output of system (1) is accessible at discrete instants of time, resulting in a sequence of $m$ dimensional vectors $y_{k}, k \in \mathbb{Z}_{\geq 1}$ defined as:

$$
y_{k}:=C x\left(t_{k}\right) \text {, }
$$

where $C \in \mathbb{R}^{m \times n}$ is full row rank and $t_{k}, k \in \mathbb{Z}_{\geq 1}$, is a sequence of increasing non-negative real numbers that satisfies the following assumption:

Assumption 1: There exist scalars $T_{m}$ and $T_{M}$, with $0<T_{m} \leq T_{M}$, such that:

$$
T_{m} \leq\left|t_{k+1}-t_{k}\right| \leq T_{M}, \quad \forall k \in \mathbb{Z}_{\geq 1}
$$

Assumption 1 considers the case of asynchronous discrete-time measurements with a sampling interval lower and upper bounded by two known positive constants $T_{m}$ and $T_{M}$. Note that $T_{m}$ must be greater than zero to avoid Zeno behaviors in the hybrid model developed below.

Taking inspiration from the hybrid systems formalism of [12], it is possible to represent the sampled-data system associated with this setting as follows:

$$
\begin{aligned}
& \left\{\begin{array}{l}
\dot{x}=A x+B u, \\
\dot{\tau}=1,
\end{array} \quad(x, \tau) \in \mathcal{C}_{x}:=\mathbb{R}^{n} \times\left[0, T_{M}\right],\right. \\
& \left\{\begin{array}{l}
x^{+}=x, \\
\tau^{+}=0,
\end{array} \quad(\hat{x}, \tau) \in \mathcal{D}_{x}:=\mathbb{R}^{n} \times\left[T_{m}, T_{M}\right],\right. \\
& y=C x,
\end{aligned}
$$

where the variable $\tau$ is a timer keeping track of the elapsed time since the last sample, and the impulsive nature of the available measurement is represented by the extra property that output $y$ is only available at jump times. With model (4), one readily sees that for any sequence $y_{k}$ in (2), satisfying (3), there exists a solution to (4) such that $y_{k}=y\left(t_{k}, k\right), k \in \mathbb{Z}_{\geq 1}$, and viceversa any solution to (4) has jump time satisfying (3).

Constraining the jump set to be included in the set where $\tau \in\left[T_{m}, T_{M}\right]$ ensures that Assumption 1 is verified as clarified in the next statement, which is a corollary of [5, Props $1.1 \& 1.2$, page 747].

Proposition 1: Consider any solution to (4) and denote by $t_{k}, k \in \mathbb{Z}_{\geq 1}$, its jump times. Then the sequence $t_{k}$ satisfies Assumption 1. Moreover, consider any sequence $t_{k}, k \in \mathbb{Z}_{\geq 1}$, satisfying Assumption 1. For each $x_{0} \in \mathbb{R}^{n}$ there exists $\tau_{0} \in\left[0, T_{M}\right]$ such that a solution $\phi$ to (4) with $\phi(0,0)=\left(x_{0}, \tau_{0}\right)$ has jump times coinciding with $t_{k}, k \in \mathbb{Z}_{\geq 1}$.

In this paper we propose an observer whose structure implicitly complies with the restriction specified in Assumption 1 on the available output. Our observer is capable of providing an asymptotic estimate of the plant state, regardless of the sequence of times $t_{k}$ at which the sampled output is available. The hybrid structure of the proposed observer is the following:

$$
\begin{cases}\dot{\hat{x}}=A \hat{x}+B u, & (\hat{x}, x, \tau) \in \mathbb{R}^{n} \times \mathcal{C}_{x}, \\ \hat{x}^{+}=\hat{x}+K(\tau)(y-C \hat{x}), & (\hat{x}, x, \tau) \in \mathbb{R}^{n} \times \mathcal{D}_{x},\end{cases}
$$

where the matrix function $K:\left[T_{m}, T_{M}\right] \rightarrow \mathbb{R}^{n \times m}$ corresponds to the time-varying gain of the observer responsible for the discrete output injection term. It is clear that, with dynamics (5), and due to Proposition 1, output $y$ is only used at the sampling instants $t_{k}$ compliant with Assumption 1.

The design of the time-varying gain $K(\cdot)$ will be performed in the next section. Note that as compared to a standard LTI Luenberger architecture (such as the one used in [10]), observer (5) is based on an injection term that depends on the elapsed time since the last measurement. This time is known to the observer by way of state $\tau$ in (4).

\section{MAIN RESUltS}

The main goal of this work is to give design rules to select the gain function $K(\cdot)$ in (5) such that the estimation error $e:=x-\hat{x}$ converges asymptotically to zero. Such a property is well characterized in terms of the stability of the following error dynamics, issued from (4)-(5):

$$
\begin{aligned}
& \left\{\begin{array}{l}
\dot{e}=A e, \\
\dot{\tau}=1,
\end{array} \quad(e, \tau) \in \mathcal{C}:=\mathbb{R}^{n} \times\left[0, T_{M}\right],\right. \\
& \left\{\begin{array}{l}
e^{+}=(I-K(\tau) C) e, \\
\tau^{+}=0,
\end{array}\right.
\end{aligned}
$$

We first present an analysis result certifying asymptotic stability of the compact set:

$$
\mathcal{A}:=\left\{(e, \tau): e=0, \tau \in\left[0, T_{M}\right]\right\}
$$


corresponding to the set where the estimation error is zero.

Note that Lemma 1 below is an extension of [10, Theorem 1] to the case of time-varying injection gain $K(\cdot)$.

Lemma 1: Assume that there exists a matrix $P=P^{\top}>0$, and a continuous matrix function $\tau \rightarrow K(\tau)$ such that:

$$
\left[\begin{array}{cc}
\mathrm{e}^{\left(-A^{\top} \tau\right)} P \mathrm{e}^{(-A \tau)} & P \\
P & P
\end{array}\right]>\left[\begin{array}{cc}
0 & \star \\
P K(\tau) C & 0
\end{array}\right], \forall \tau \in\left[T_{m}, T_{M}\right] .
$$

Then set $\mathcal{A}$ in (7) is uniformly globally asymptotically stable ${ }^{1}$ (UGAS) for the error dynamics in (6).

Based on the analysis result of Lemma 1, we can now prove a few relevant constructions for the gain $K(\cdot)$, corresponding to a few special cases. The first case is relatively straightfoward and corresponds to the case where $C$ is invertible (namely the state is completely accessible at the sampling instants). This case is somewhat interesting because it corresponds to the source of inspiration of the subsequent construction, and has been used in a dedicated application by the first author in [3]. It is reported below.

Theorem 1: If $C$ is invertible, then for any $P=P^{\top}>0$ and any $\lambda \in(-1,1)$, inequality (8) is satisfied with:

$$
K(\tau)=\left(I-\lambda \mathrm{e}^{(-A \tau)}\right) C^{-1},
$$

which then guarantees UGAS of $\mathcal{A}$ for system (6).

Algorithm 1 A nUMERICAL PROCEDURe to SOLVE (11)

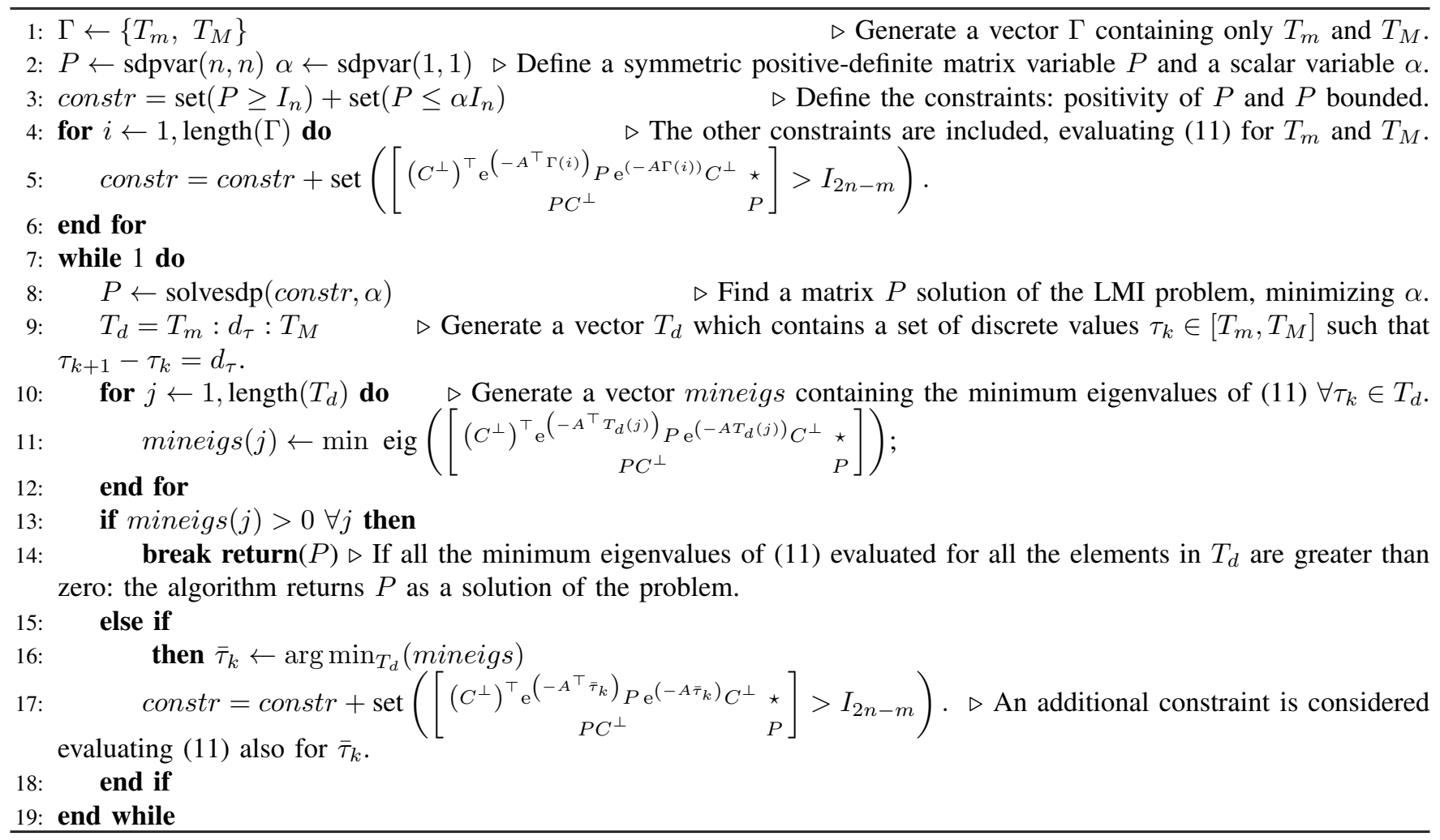

Remark 1: The effect of selection (9) in Theorem 1 on the jump equation (6b) of the error dynamics is insightful in terms of the selection of constant $\lambda$ in (9). In particular, using (9) in (6b), we obtain:

$$
e^{+}=\lambda \mathrm{e}^{(-A \tau)} e,
$$

which clearly reveals that the choice $\lambda=0$ leads to a dead-beat controller, while the choice $\lambda= \pm 1$ leads to a nontrivial reset that resets back the estimation error to the value that it had immediately after the previous sample (this fact is evident by keeping in mind the explicit expression of the error $e(t, k)=\mathrm{e}^{(A \tau)} e\left(t_{k}, k\right)$ for all $\left.t \in\left[t_{k}, t_{k+1}\right]\right)$. Clearly, the choice $\lambda= \pm 1$ is not allowed in our result because it leads to bounded, but non converging, responses.

The solution of Theorem 1 is only viable under demanding conditions on the available measurements, which are only seldom verified. Due to this reason, one of the main contributions of this paper is the result given next, which provides a

${ }^{1}$ For a definition of uniform global asymptotic stability of a compact attractor for a hybrid system, see [12, Ch 3]. 
construction for the gain $K(\cdot)$ as long as one can find a constant matrix $P$ satisfying the following infinite set of matrix inequalities:

$$
\Xi_{P}(\tau):=\left[\begin{array}{cc}
\left(C^{\perp}\right)^{\top} \mathrm{e}^{\left(-A^{\top} \tau\right)} P \mathrm{e}^{(-A \tau)} C^{\perp} & \star \\
P C^{\perp} & P
\end{array}\right]>0,
$$

$$
\forall \tau \in\left[T_{m}, T_{M}\right],
$$

where $C^{\perp}$ denotes the orthogonal complement of $C^{\top}$, namely a matrix such that $\left[C^{\perp} C^{\top}\right]$ is square and nonsingular, and such that $C C^{\perp}=0$.

Matrix inequality (11) is not easy to solve, but we discuss in Section IV a numerical algorithm that is able to perform this task. We report below the explicit expression of $K(\cdot)$, which induces UGAS of attractor $\mathcal{A}$ for the observation error dynamics, as long as (11) is satisfied.

Theorem 2: Assume that $C$ is full row rank and denote by $C^{\perp}$ a basis of the orthogonal complement of $C^{\top}$. If there exists $P=P^{\top}>0$ satisfying (11), then selection:

$$
\begin{array}{r}
K(\tau):=\left(C^{\top}-C^{\perp}\left(\left(C^{\perp}\right)^{\top} \mathrm{e}^{\left(-A^{\top} \tau\right)} P \mathrm{e}^{(-A \tau)} C^{\perp}\right)^{-1}\right. \\
\left.\left(C \mathrm{e}^{\left(-A^{\top} \tau\right)} P \mathrm{e}^{(-A \tau)} C^{\perp}\right)^{\top}\right)\left(C C^{\top}\right)^{-1}
\end{array}
$$

guarantees UGAS of $\mathcal{A}$ for system (6).
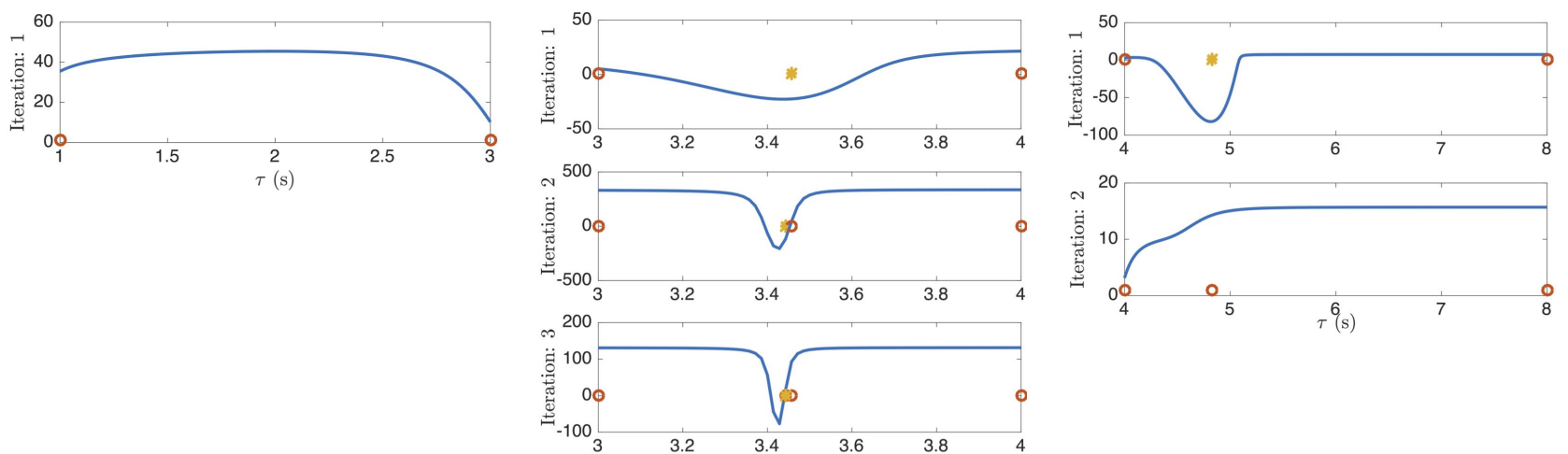

(a)

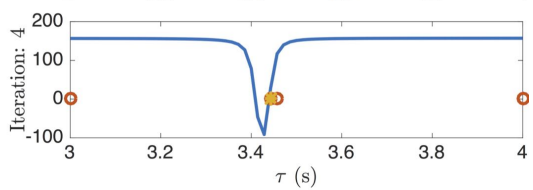

(b)

(c)

Fig. 1. Minimum eigenvalues of matrix (11) for $\tau \in[1,3]$ in (a), for $\tau \in[3,4]$ in (b) and for $\tau \in[4,8]$ in (c).

\section{NUMERICAL ALGORITHM}

In this section we introduce a numerical algorithm, aiming to find a matrix $P$ satisfying the matrix inequality (11) $\forall \tau \in\left[T_{m}, T_{M}\right]$. The scheme of the algorithm is shown at the top of the next page. For a given system (1)-(2), a vector $\Gamma$ is defined, which contains only the values $T_{m}$ and $T_{M}$. Then in the first step an LMI problem is solved considering as constraints the positivity of $P$ and the inequality (11) evaluated for all the elements of $\Gamma$ ( $T_{m}$ and $T_{M}$ in the first step). An upper bound on $P$ has been introduced, $P \leq \alpha I_{n}$, in order to avoid solutions that lead to large values of $P$ and consequently to an aggressive dynamics of the observer. For numerical reasons, we constrain our bounds to be larger than $I$ rather than zero, because this does not affect the feasibility properties from homogeneity of the LMI conditions.

Then there is a check phase. A vector $T_{d}$ is generated and it contains a set of discrete values $\tau_{k} \in\left[T_{m}, T_{M}\right]$ such that: $\tau_{k+1}-\tau_{k}=d_{\tau} \ll\left|T_{M}-T_{m}\right|$. For each $\tau_{k}$ the eigenvalues of matrix (11) are computed and a vector containing all the minimum eigenvalues is populated. If all the elements of this vector are greater than zero, the algorithm is stopped and the solution $P$ is given. Otherwise, the value $\bar{\tau}_{k}$ which gives the minimum negative eigenvalue is defined, and an additional constraint is considered, evaluating (11) also for $\bar{\tau}_{k}$. Obviously, at the second step the LMI will be solved considering the inequality (11) computed with $\tau$ equal to $T_{m}, T_{M}$ and the new value $\bar{\tau}_{k}$, and so on. The algorithm stops when all the minimum eigenvalues computed in the check phase are positive, or when the problem is infeasible. If the problem is infeasible, then no solution is found. This last case can occur, for example, when the sampled plant of the system is not 


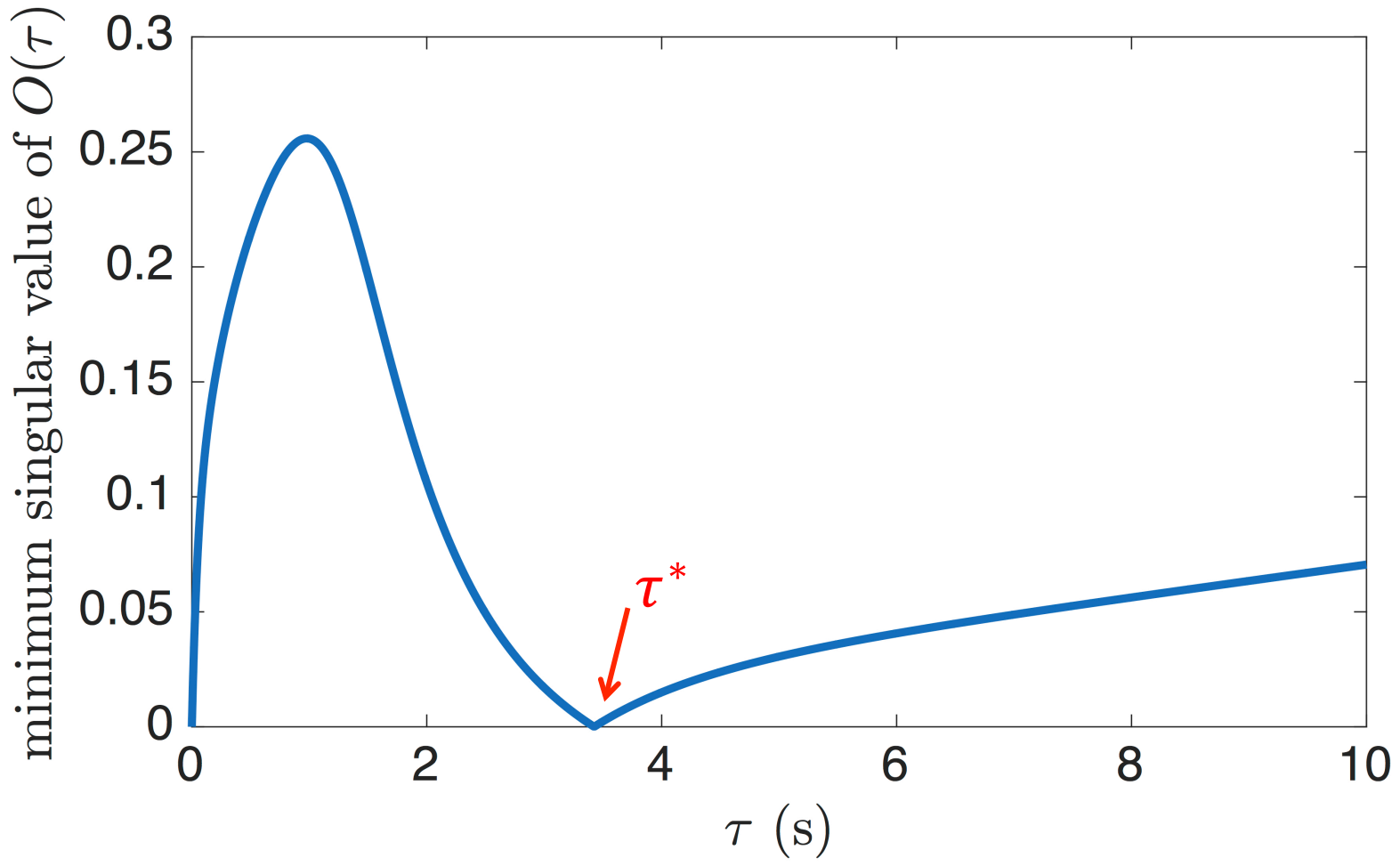

Fig. 2. Minimum singular values of the observability matrix $\mathcal{O}(\tau)$ for the periodically sampled plant.

observable for some values of $\tau$, i.e. when there exists a $\tau^{\star} \in\left[T_{m}, T_{M}\right]$ such that the minimum singular value of the matrix $\mathcal{O}(\tau)$ defined as:

$$
\mathcal{O}\left(\tau^{\star}\right)=\left[\begin{array}{llll}
C & C \mathrm{e}^{A \tau^{\star}} & \cdots & C\left(\mathrm{e}^{A \tau^{\star}}\right)^{n-1}
\end{array}\right]^{\top}
$$

is zero. In the following a numerical example is illustrated which shows the application of the proposed algorithm.

\section{A. Numerical example}

Let us consider system (1)-(2) with the following unstable plant:

$$
A=\left[\begin{array}{ccc}
-0.02 & -1.4 & 9.8 \\
-0.01 & -0.4 & 0 \\
0 & 1 & 0
\end{array}\right], B=\left[\begin{array}{c}
9.8 \\
6.3 \\
0
\end{array}\right], C=\left[\begin{array}{l}
1 \\
0 \\
1
\end{array}\right]^{\top},
$$

for which $\operatorname{eig}(A)=\{-0.656,0.118+0.368 i, 0.118-0.368 i\}$. For the matrix $C$ in (14) we have $C^{\perp}=\left[\begin{array}{ccc}0 & 1 \\ 0.7071 & 0 & -0.7071\end{array}\right]^{\top}$.

In this case the proposed algorithm is applied for three different choices of $\left[T_{m}, T_{M}\right]$. In particular for $\tau \in[1,3]$, $\tau \in[3,4]$ and $\tau \in[4,8]$.

In the first case, $\tau \in\left[T_{m}, T_{M}\right]=[1,3]$, the algorithm finds a solution with only one iteration. Indeed, at the first iteration, the algorithm considers only $\Gamma=\left\{T_{m}, T_{M}\right\}$, and, during the check phase, all the minimum eigenvalues of matrix (11) result greater than zero for all $\tau \in T_{d}$, as shown in Figure 1(a). The value of $P$, solution of the problem is:

$$
P=10^{4}\left[\begin{array}{ccc}
0.8334 & -0.0041 & 0.8333 \\
-0.0041 & 2.0116 & -80.0359 \\
0.8333 & -0.0359 & 0.8341
\end{array}\right], \quad \tau \in[1,3] .
$$

In a second case we select $\tau \in\left[T_{m}, T_{M}\right]=[3,4]$ and the algorithm does not find a solution because the periodically sampled plant is not observable for $\tau^{*}=3.425 \in[3,4]$ (namely for this $\tau^{*}$ the matrix in (13) is singular). This fact is clear looking at Figure 2 where the minimum singular values of the observability matrix $\mathcal{O}(\tau)$ are shown, and for $\tau^{*}=3.425$ the minimum singular value is zero, revealing a rank loss for the observability matrix. This fact is confirmed by Figure 1(b), where it is shown that, after four iterations, the minimum eigenvalue of matrix (11) is always negative in a neighborhood of $\tau=3.425$.

Finally, in the last case, we select $\tau \in\left[T_{m}, T_{M}\right]=[4,8]$, and the algorithm finds a solution with two iterations as shown in Figure 1(c). Indeed, during the first iteration the algorithm considers $\Gamma=\left\{T_{m}, T_{M}\right\}$, and, during the check phase, not all the minimum eigenvalues of matrix (11) result greater than zero for $\tau \in T_{d}$ (see the upper subplot of Figure 1(c)). In particular it results that the minimum negative eigenvalue is obtained at $\tau=4.84$, so an additional constraint is considered evaluating (11) for $\bar{\tau}_{k}=4.84$. In the second iteration, the algorithm finds a solution as it is evident from the lower subplot of Figure 1(c). The value of $P$, solution of the problem is:

$$
P=10^{4}\left[\begin{array}{ccc}
0.9573 & -0.0031 & 0.9571 \\
-0.0031 & 2.2754 & -0.0122 \\
0.9571 & -0.0122 & 0.9573
\end{array}\right], \quad \tau \in[4,8] .
$$




\section{Simulation Results}

The proposed observer has been tested by means of numerical simulations and the results are given in the following. Initially, the unstable plant (14) has been stabilized by means of a state feedback using a low gain $K_{u}=10^{-2}[0.165 .47-$ 0.01], such that $\operatorname{eig}\left(A+B K_{u}\right)=\{-0.01,-0.02,-0.03\}$. The corresponding slow transient ensures that the signals do not blow up during the simulation, but they are associated to a sufficiently rich behavior.

The proposed observer has been tested in two different conditions considering the two cases described in Section IV-A, with $\tau \in[1,3]$ and $\tau \in[4,8]$.

The dynamics expressed in (4) has been implemented together with the observer (5) in the MATLAB ${ }^{\circledR}$-Simulink environment. The gain $K(\tau)$ is computed on-line according to (12) by using matrix $P$ in (15) for $\tau \in[1,3]$ and $P$ in (16) for $\tau \in[4,8]$. Moreover, in order to implement a random value of the time-instant of the measurements, we implement the following modified error dynamics, corresponding to (6) with random selection of the inter-measurement intervals:

$$
\begin{aligned}
& \left\{\begin{array}{l}
\dot{e}=A e, \\
\dot{\tau}=1, \\
\dot{\tau}_{r}=-1,
\end{array} \tau_{r} \in\left[T_{M}, 0\right],\right. \\
& \left\{\begin{array}{l}
e^{+}=(I-K(\tau) C) e, \\
\tau^{+}=0, \\
\tau_{r}^{+}=T_{m}+\left(T_{M}-T_{m}\right) \nu^{+},
\end{array} \quad \tau_{r}=0 .\right.
\end{aligned}
$$

where $\nu^{+}$is a random variable uniformly distributed in the interval $[0,1]$. Note that this modified dynamics is represented with the notation in [6].

In Figure 3 the real and estimated state vector components $x_{i}, \hat{x}_{i}, i=1,2,3$, as well as estimation errors $e_{i}=x_{i}-\hat{x}_{i}$, $i=1,2,3$, are shown, during the first test with $\tau \in[1,3]$. Moreover, for the same test, the waveforms of the Lyapunov function $V$ (See [22]), of the variables $\tau$ and $\tau_{r}$ and of the output error $y-\hat{y}$ are shown in Figure 4.

From Figure 3 it is evident that the estimated variables track very well the corresponding state variables and all the errors go to zero asymptotically. Moreover, it is possible to note the impulsive behavior of the estimate especially during the initial transient. From Figure 4 we note that the Lyapunov function is constant during flow, and decreases across jumps, as expected from the theoretical results reported in [22]. Finally, from the waveforms of $\tau$ and $\tau_{r}$ we see that the jumps occur randomly in the interval $[1,3]$ according to the described dynamics (17).

Figures 5-6 show the results for the same test described above, but when the measurements are provided more sporadically, $\tau \in[4,8]$. In this case the same comments given for the first test can be provided, confirming the effectiveness of the proposed approach. Obviously, the convergence rate in this case is slower because the measurements are accessible less frequently.

\section{CONCLUSION}

In this work a time-varying observer for a linear continuous-time plant with asynchronous discrete-time measurements has been proposed. In particular some theoretical tools have been provided, in terms of LMIs, certifying asymptotic stability of a certain compact set where the estimation error is zero. The developed solutions are formalized using a hybrid systems framework, thus providing an elegant setting. Two solutions have been proposed, one under the restrictive assumption that the output matrix is invertible, and one for the more general case of a detectable pair, under the assumption that some LMI conditions hold. The results provided by a numerical example show the effectiveness of the proposed approach, confirming the theoretical results and the feasibility of the proposed numerical solution.

\section{REFERENCES}

[1] T. Ahmed-Ali, I. Karafyllis, and F. Lamnabhi-Lagarrigue. Global exponential sampled-data observers for nonlinear systems with delayed measurements. Systems \& Control Letters, 62(7):539-549, 2013.

[2] T. Ahmed-Ali, V. Van Assche, J. Massieu, and P. Dorleans. Continuous-discrete observer for state affine systems with sampled and delayed measurements. IEEE Transactions on Automatic Control, 58(4):1085-1091, 2013.

[3] F. Alonge, F. D'Ippolito, A. Gargano, and A. Sferlazza. Hybrid nonlinear observer for inertial navigation. In IEEE International Symposium on Industrial Electronics, pages 381-386. IEEE, 2016.

[4] V. Andrieu and M. Nadri. Observer design for Lipschitz systems with discrete-time measurements. In IEEE Conference on Decision and Control, pages $6522-6527$.

[5] C. Cai, A. Teel, and R. Goebel. Smooth Lyapunov functions for hybrid systems Part II:(pre) asymptotically stable compact sets. IEEE Transactions on Automatic Control, 53(3):734-748, 2008.

[6] C. Cai and A. R. Teel. Robust input-to-state stability for hybrid systems. SIAM Journal on Control and Optimization, 51(2):1651-1678, 2013.

[7] T. Chen and B. A. Francis. Optimal sampled-data control systems. Springer Science \& Business Media, 2012.

[8] T. N. Dinh, V. Andrieu, M. Nadri, and U. Serres. Continuous-discrete time observer design for lipschitz systems with sampled measurements. IEEE Transactions on Automatic Control, 60(3):787-792, 2015.

[9] M. Farza, M. M'Saad, M. L. Fall, E. Pigeon, O. Gehan, and K. Busawon. Continuous-discrete time observers for a class of mimo nonlinear systems. IEEE Transactions on Automatic Control, 59(4):1060-1065, 2014. 
[10] F. Ferrante, F. Gouaisbaut, R. G. Sanfelice, and S. Tarbouriech. State estimation of linear systems in the presence of sporadic measurements. Automatica, 73:101-109, 2016.

[11] F. Ferrante, F. Gouaisbaut, and S. Tarbouriech. Stabilization of continuous-time linear systems subject to input quantization. Automatica, 58:167-172, 2015.

[12] R. Goebel, R. G. Sanfelice, and A. R. Teel. Hybrid Dynamical Systems: modeling, stability, and robustness. Princeton University Press, 2012.

[13] J. P. Hespanha, P. Naghshtabrizi, and Y. Xu. A survey of recent results in networked control systems. Proceedings of the IEEE, 95(1):138-162, 2007.

[14] S. Kluge, K. Reif, and M. Brokate. Stochastic stability of the extended Kalman filter with intermittent observations. IEEE Transactions on Automatic Control, 55(2):514-518, 2010.

[15] Y. Li, S. Phillips, and R. Sanfelice. On distributed observers for linear time-invariant systems under intermittent information constraints. In IFAC Symposium on Nonlinear Control Systems. IFAC, 2016.

[16] D. Liberzon. Switching in systems and control. Springer Science \& Business Media, 2012.

[17] M. Nadri, H. Hammouri, and R. M. Grajales. Observer design for uniformly observable systems with sampled measurements. IEEE Transactions on Automatic Control, 58(3):757-762, 2013

[18] K. Plarre and F. Bullo. On Kalman filtering for detectable systems with intermittent observations. IEEE Transactions on Automatic Control, 54(2):386-390, 2009.

[19] T. Raff and F. Allgöwer. An impulsive observer that estimates the exact state of a linear continuous-time system in predetermined finite time. In Mediterranean Conference on Control \& Automation, pages 1-3, 2007.

[20] T. Raff, M. Kögel, and F. Allgöwer. Observer with sample-and-hold updating for lipschitz nonlinear systems with nonuniformly sampled measurements. In American Control Conference, 2008, pages 5254-5257. IEEE, 2008.

[21] E. R. Rohr, D. Marelli, and M. Fu. Kalman filtering with intermittent observations: On the boundedness of the expected error covariance. IEEE Transactions on Automatic Control, 59(10):2724-2738, 2014.

[22] A. Sferlazza, S. Tarbouriech, and L. Zaccarian. Time-varying sampled-data observer with asynchronous measurements (long version). Available at https://hal.archives-ouvertes.fr/hal-01488636, pages 1-14, 2017.

[23] B. Sinopoli, L. Schenato, M. Franceschetti, K. Poolla, M. Jordan, and S. S. Sastry. Kalman filtering with intermittent observations. IEEE Transactions on Automatic Control, 49(9):1453-1464, 2004. 

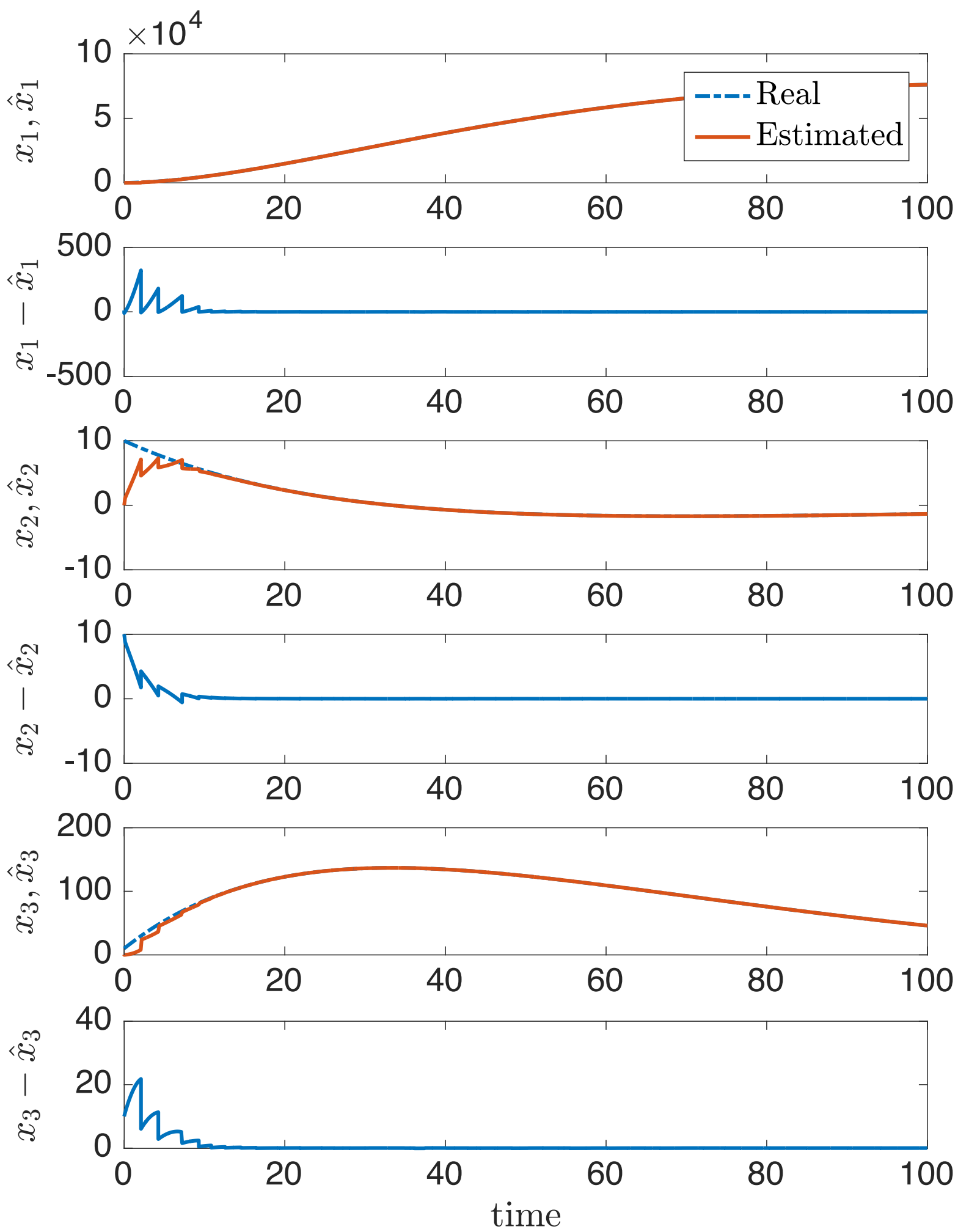

Fig. 3. Real and estimated state vector components $x_{i}, \hat{x}_{i}, i=1,2,3$, as well as estimation errors $x_{i}-\hat{x}_{i}, i=1,2,3$, during a test with $\tau \in[1,3]$. 

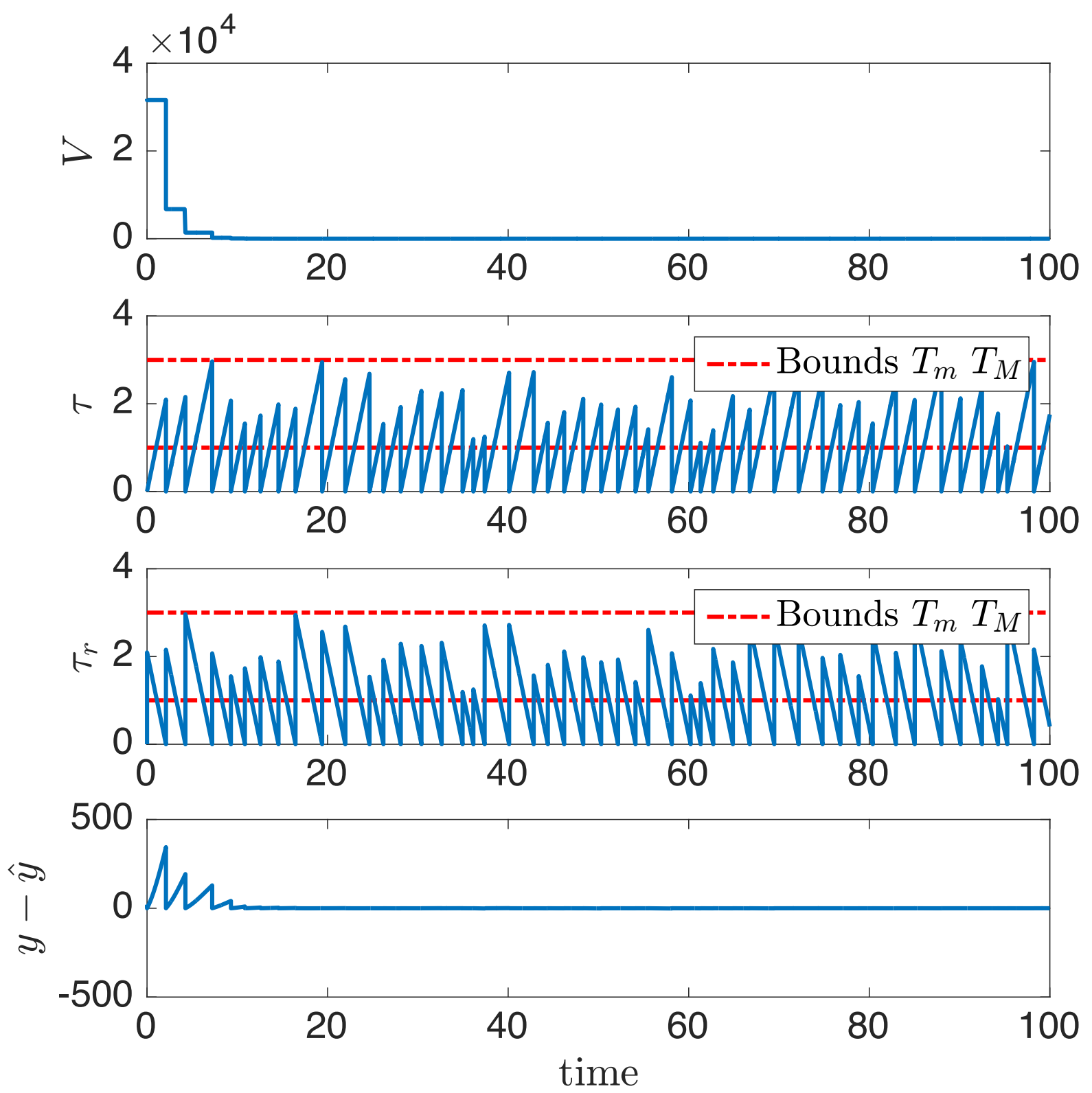

Fig. 4. Waveforms of the Lyapunov function $V$, of the variables $\tau$ and $\tau_{r}$ and of the output error $y-\hat{y}$, during a test with $\tau \in[1,3]$. 

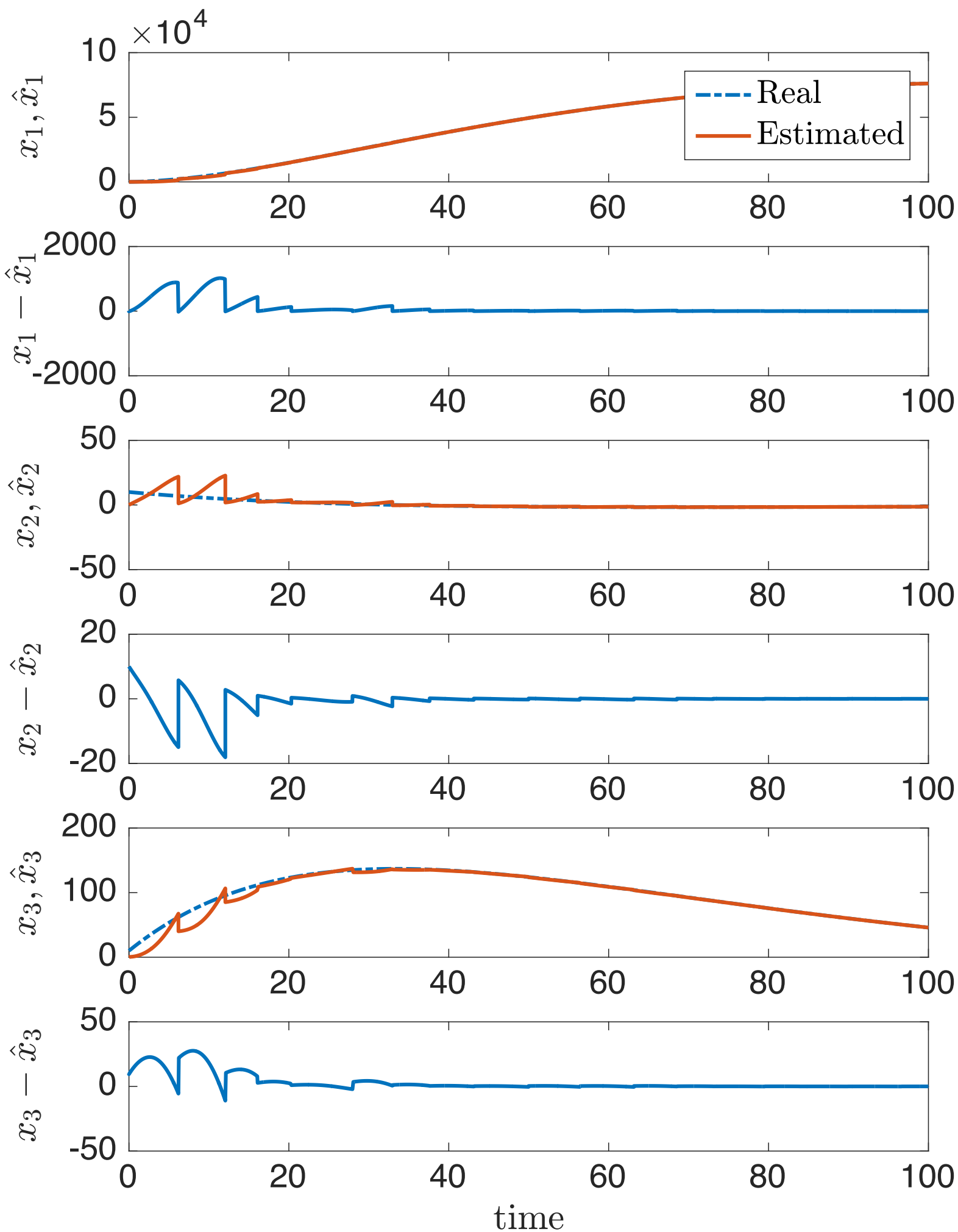

Fig. 5. Real and estimated state vector components $x_{i}, \hat{x}_{i}, i=1,2,3$, as well as estimation errors $x_{i}-\hat{x}_{i}, i=1,2,3$, during a test with $\tau \in[4,8]$. 

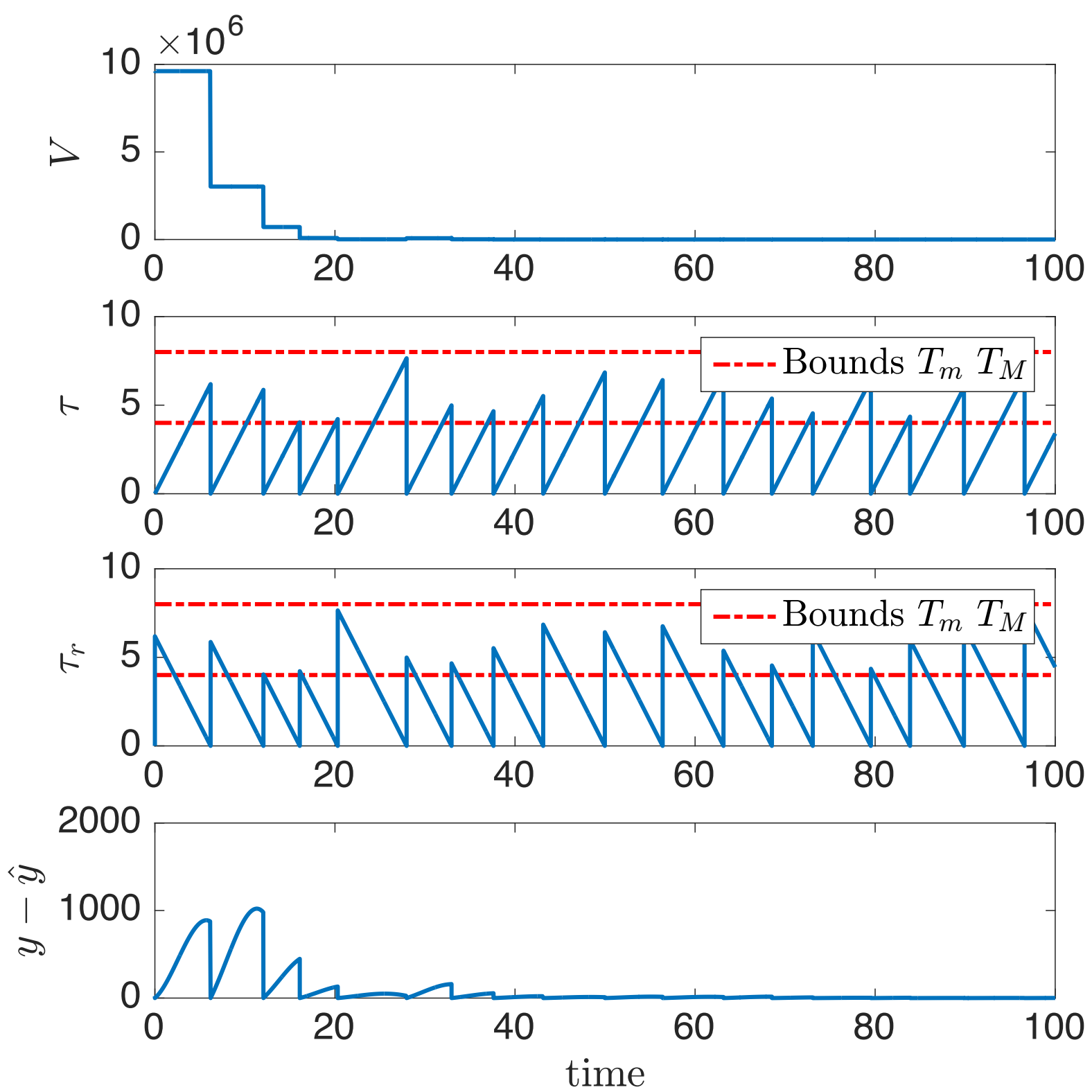

Fig. 6. Waveforms of the Lyapunov function $V$, of the variables $\tau$ and $\tau_{r}$ and of the output error $y-\hat{y}$, during a test with $\tau \in[4,8]$. 